\title{
Does Research Improve Patient Care?
}

You can have data without information, but you cannot have information without data.

—Daniel Keys Moran, American Science Fiction Writer (1962-)

\subsection{Are Patient Care and Clinical Research Related Activities?}

Patient care and research are complementary. If you do research it helps you to become a better clinician. The first step in doing clinical research is to formulate a research question [1]. The sequence of events which helps in studying gaps in knowledge comes from regularly reading published scientific papers which in turn are based upon the data generated by others. Patients are living teachers and each one teaches us something new.

The mind of a clinician should always be curious to know what the deficiencies are in current knowledge while pursuing practice. A major excitement in medicine is the working up of undiagnosed cases. These cases are like solving a jigsaw puzzle where the physician puts in each piece of evidence to make a pattern and solve a mystery. Another challenging area is to treat patients who have 'grey' illnesses, i.e., those whose management has not been described in any published books or guidelines. 


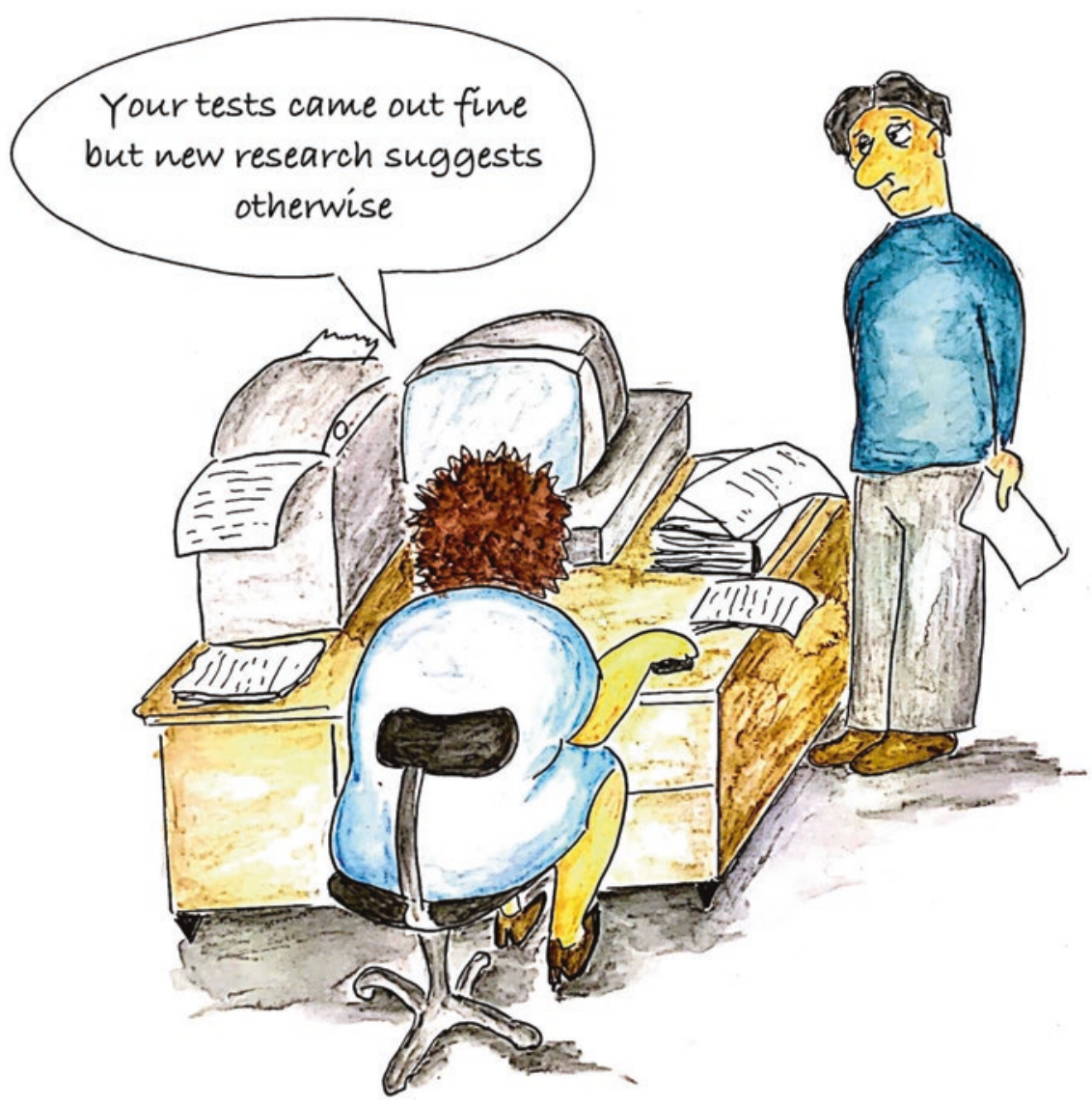

\subsection{How Can One Blend Research with Practice?}

Practicing physicians have been involved in clinical research for many years. It is usually said that 'The gap between research and health care practice can be reduced by the immersion of the researcher in the practice environment' [2]. Initially, research was limited to collecting data or performing retrospective analyses of such data and describing case reports or case series. Recently, scientific techniques have exploded with many new types of study design, such are randomized controlled trials, metanalyses, quality of life investigations, and translational medicine research. The average doctor needs to be taught how to carry out research as this is usually not covered in their curriculum. He needs to be trained how to write research protocols, what the types of study design are, how analyses including metanalyses are done, and basic statistics. Till he/she receives such hands-on training on how to do research he/she may have to just limit research activity to gathering retrospective data and not venture into new and exciting research paths. 


\subsection{How Should I Start Research in My Institution?}

You first need to build synergy between your clinical and research teams. The clinical team should have a consultant and associates, residents, and nurses whereas the research team should include a research coordinator and a statistician. You will also require a computer and Internet access. Finally, you must have the will to persist till you reach your goal and then to write up the results well. You may not start by being proficient in medical writing but this skill can be developed over time by sheer persistence. Another key principle is to find a good and helpful mentor who can help you navigate through this exciting journey.

\subsection{What is Evidence-based Medicine?}

Evidence-based medicine is the meticulous, clear, judicious, and reasonable use of current, best evidence in making clinical decisions about the care of individual subjects. Evidence-based medicine integrates clinical experience, scientific information, and patients' values for treating a disease [3] (Fig. 5.1).

The concept of evidence-based medicine is not new. The first record of this is from the year 1192-1250 AD, when the Emperor Frederick II who ruled Rome, Sicily, and Jerusalem showed his interest in this type of experiment. He showed the effect of exercise on digestion using the knights. He gave them identical meals and then one was sent for hunting and the other was restricted to bed. Several hours later he killed both of them to examine the residue in the gastrointestinal tract. The inactive one had the greater stomach residue. The experiment was evidence based but definitely not a good example to emulate!

The effect of bloodletting on humans was also studied based on a randomized trial by Jan Baptista Van Helmont in the seventeenth century. He divided the poor

Fig. 5.1 Evidence-based medicine: integration of clinical experience, patient values, and expectations and best external evidence

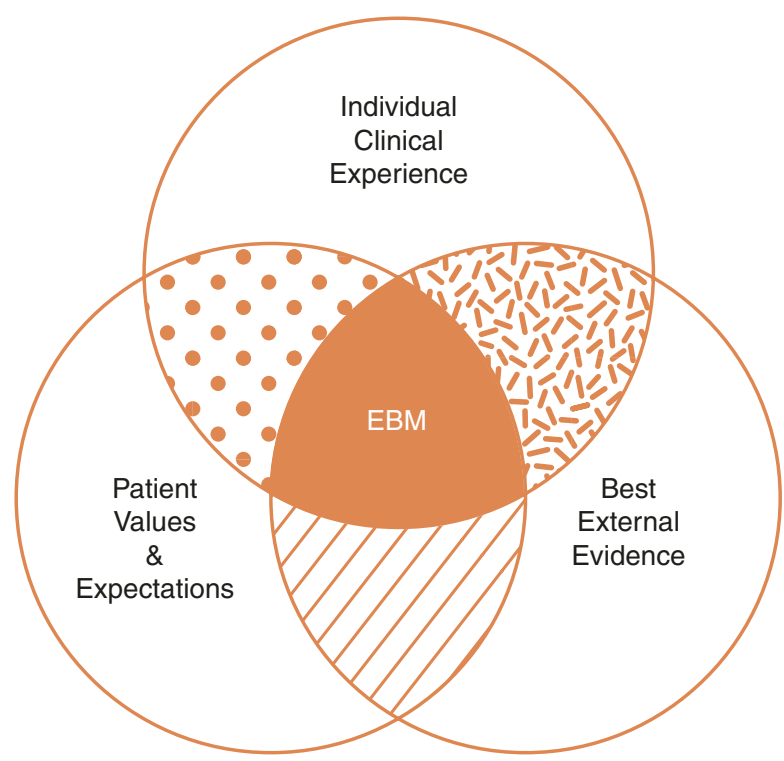


population into sample sizes between 200 and 500 by casting lots. While one group had a phlebotomy, the other was left alone. The number of funerals was recorded in both the groups. However, in the nineteenth century when Pierre Charles Alexendrae Louis introduced the concept of statistical analysis for the treatment of medical outcomes he showed that bloodletting did not change the outcome of disease. Despite this the practice of bloodletting did not change for many years.

James Lind, a Scottish physician will be given the credit for conducting the first documented experimental trial in medical history, by administrating citrus fruits to treat scurvy. He conducted the trial on a naval ship in 1747 by giving oranges and limes to half of the sailors affected by scurvy and not to the others, and recorded their progress and outcomes in detail. Those who were given citrus fruits recovered from scurvy and those who were given did not. Lind concluded that there was something in the fruit (later shown to be Vitamin C), which prevented scurvy [4].

Now the Cochrane Library synthesizes the best evidence on individual medical problems and publishes authoritative and widely read systematic reviews and summaries of the results $[5,6]$.

\subsection{How Can Research Data Be Incorporated into Clinical Practice?}

Previously reading medical journals used to involve going to library and reading printed papers. However, this has now changed tremendously with the advent of the Internet and most information is widely and easily available through laptop, computers, iPads, and even smartphones. This has revolutionized medical education and research and we will discuss this in detail in a subsequent chapter [7].

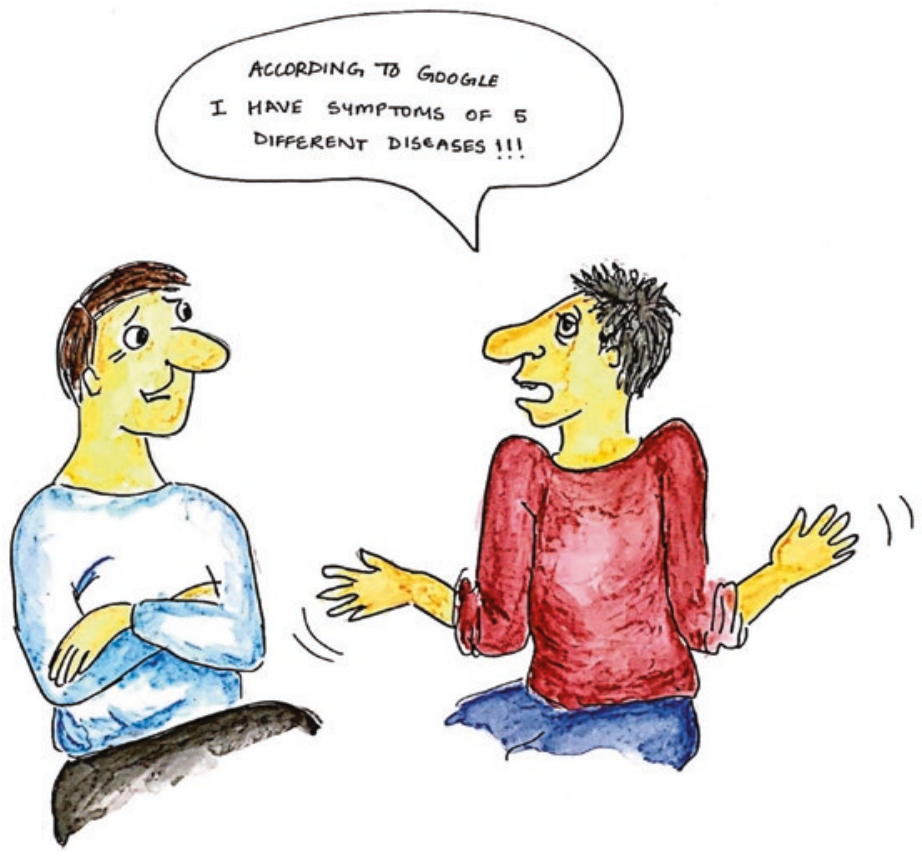




\subsection{What are the Limitations of Medical Care in Developing Countries?}

The expenditure on health care in developing countries is very small. There are many reasons for this but their low Gross Domestic Product (GDP) is probably the single most important factor. Most developing nations spend about $1-2 \%$ of their GDP on health care compared with the USA that spends $18 \%$ of a much larger GDP [8]. What is expected from us in our resource-constrained settings is to provide a high quality of health care to our population, which is accessible, affordable, and appropriate. For this we need to be guided by evidence on how to do this which is relevant to us and this can only be obtained by doing research into our own problems.

\subsection{How Can Commonly Available Data Help in Research?}

Developing countries generally have large populations. If we collect basic data on the common diseases, we will find them to be very different compared to those in the Western countries. Data collection on a common condition like hypertension has helped us realize its prevalence in a younger age group here $[9,10]$, diabetes mellitus type 2 in India is associated with a low BMI [11]. The common sites of cancers are in the mouth and cervix $[12,13]$, and rheumatoid arthritis is more common than we previously thought [14]. Using this data, we can plan strategies for diagnosis, treatment, and prevention.

\subsection{What is Health Outcomes Research?}

Health outcome studies are those which link the treatment or intervention given to a patient and its end result (outcome) (Fig. 5.2) [15]. These studies let us know what works and what does not. These studies can easily be done from good medical

Fig. 5.2 Factors affecting health outcomes

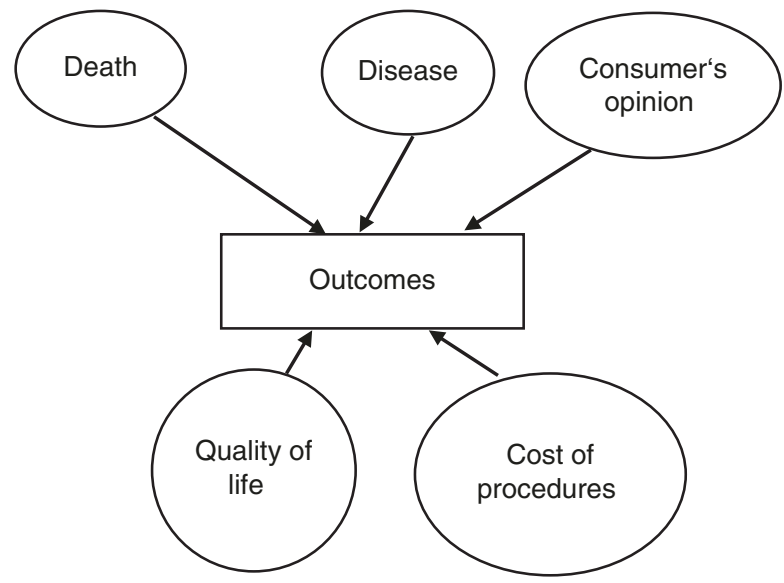


records, clinical audit, and the databases of insurance companies. They can guide us towards correct interventions and decisions, to compare whether the medical facilities available match performance and can help uncover deficiencies. These studies are on safety, efficacy, effectiveness, equality, timeliness, system responsiveness, and are patient centric.

\subsection{How is Research Perceived among Doctors?}

The way we perceive research has also to be changed. It is usually thought that research is 'experimental and dangerous' for patients. It's safety aspects and patientcentric approach need to be highlighted. The benefits of the research can be exemplified by the following. A large study was conducted in England between 2001 and 2008 involving more than 200,000 patients with colorectal cancer. In patients who participated in the trial the post-operative mortality was significantly less $(p<0.001)$ and also there was improved survival $(p<0.001)$ than in those who did not. Another interesting finding is that even patients who are not enrolled in clinical trials had better outcomes in hospitals that are active in research than in those who are not [16]. In another systematic review and metanalysis on women's health issues of 11 obstetric and 10 gynaecological studies, participation in a clinical trial consistently improved patient outcomes [17].

\subsection{Conclusions}

- Research and patient care are related activities. Increasing research involvement improves patient care.

- Simple data collection can lead to us to discover how we might help patients better.

- It is easy to blend patient care and research by learning simple research methodologies.

- Health outcomes research measures the value of a particular course of therapy and helps in decision-making.

- Practicing evidence-based medicine improves the quality of treatment patients receive and the most competent clinicians are those who keep abreast of what is the best current evidence by comprehensive and systematic literature searches while treating their patients.

\section{References}

1. Katzka DA. How to balance clinical work and research in the current era of academic medicine. Gastroenterology. 2017;153:1177-80.

2. Kajamaa A, de la Croix A, Mattick K. How to... use qualitative research to change practice. Clin Teach. 2019 Oct;16(5):437-41.

3. Masic I, Miokovic M, Muhamedagic B. Evidence based medicine-new approaches and challenges. Acta Inform Med. 2008;16:219-25. 
4. Lind J. Wikipedia. Available on https://en.wikipedia.org/wiki/James_Lind. Accessed 10 May 2020.

5. Alper BS, Hand JA, Elliott SG, Kinkade S, Hauan MJ, Onion DK, Sklar BM. How much effort is needed to keep up with the literature relevant for primary care? J Med Libr Assoc. 2004 Oct;92(4):429.

6. Chchrane Library. Available on https://www.cochranelibrary.com. Accessed 10 May 2020.

7. Baumgart DC, Wende I, Grittner U. Tablet computer enhanced training improves internal medicine exam performance. PLoS One. 2017;12:e0172827.

8. Ashraf QH, Lester A, Weil DN. When does improving health raise GDP? NBER Macroecon Annu. 2009;23:157-204.

9. Jose AP, Prabhakaran D. World Hypertension Day: contemporary issues faced in India. Indian J Med Res. 2019;149:567-70.

10. Anchala R, Kannuri NK, Pant H, Khan H, Franco OH, Di Angelantonio E, Prabhakaran D. Hypertension in India: a systematic review and meta-analysis of prevalence, awareness, and control of hypertension. J Hypertens. 2014 Jun;32(6):1170.

11. Dutta D, Ghosh S. Young-onset diabetes: an Indian perspective. Indian J Med Res. 2019;149:441-2.

12. Gupta N, Chitalkar P, Mishra R, Punia A. Epidemiology of cancer in young in central India: an analysis of rural cancer hospital data. South Asian J Cancer. 2017;6(4):183-5.

13. Kakkar N, Gupta A, Sharma NK, Agarwal P, Kaur J. Adolescents and young adults: a study of distribution of cancer at ages 15-39 years in a tertiary care hospital from North India: epidemiological considerations. South Asian J Cancer. 2017;6:180-2.

14. Handa R, Rao URK, Lewis JFM, Rambhad G, Shiff S, Ghia CJ. Literature review of rheumatoid arthritis in India. Int J Rheum Dis. 2015;19:440-51.

15. Stewart MG. Outcomes research: an overview. ORL J Otorhinolaryngol Relat Spec. 2004;66:163-6.

16. Downing A, Morris EJ, Corrigan N, Sebag-Montefiore D, Finan PJ, Thomas JD, Chapman M, Hamilton R, Campbell H, Cameron D, Kaplan R. High hospital research participation and improved colorectal cancer survival outcomes: a population-based study. Gut. 2017 Jan $1 ; 66(1): 89-96$.

17. Nijjar SK, D’Amico MI, Wimalaweera NA, Cooper N, Zamora J, Khan KS. Participation in clinical trials improves outcomes in women's health: a systematic review and meta-analysis. BJOG. 2017;124:863-71.

Open Access This chapter is licensed under the terms of the Creative Commons Attribution 4.0 International License (http://creativecommons.org/licenses/by/4.0/), which permits use, sharing, adaptation, distribution and reproduction in any medium or format, as long as you give appropriate credit to the original author(s) and the source, provide a link to the Creative Commons license and indicate if changes were made.

The images or other third party material in this chapter are included in the chapter's Creative Commons license, unless indicated otherwise in a credit line to the material. If material is not included in the chapter's Creative Commons license and your intended use is not permitted by statutory regulation or exceeds the permitted use, you will need to obtain permission directly from the copyright holder.

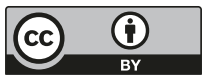

